\title{
Use of Cannabis by Young People and Psychopathological Disorders, in Fatick (Senegal)
}

\author{
Lamine Fall1, Ousmane Sy², Ndeye Diale Ndiaye-Ndongo ${ }^{3}$, Aïda Sylla ${ }^{3}$ \\ ${ }^{1}$ Department of Psychiatry, University Cheikh Anta Diop, Dakar, Senegal \\ ${ }^{2}$ Mental Health Hospital "Dalal Xel", Fatick, Senegal \\ ${ }^{3}$ Service of Psychiatry, National and Academic Hospital of Fann, Dakar, Senegal \\ Email: lamzobe@yahoo.fr
}

Received 23 January 2014; revised 21 February 2014; accepted 15 March 2014

Copyright (C) 2014 by authors and Scientific Research Publishing Inc.

This work is licensed under the Creative Commons Attribution International License (CC BY). http://creativecommons.org/licenses/by/4.0/

(c) (i) Open Access

\section{Abstract}

Background: Despite progressive increasing of drug use or drug addiction particularly with cannabis in young people, knowledge is limited about their consequences on mental health in adulthood. In Fatick (Senegal), the mental health hospital "Dalal Xel" receives many young people who present psychiatric troubles necessitating a hospitalisation. Frequently, their history reveals use or abuse of cannabis joined sometimes with other drug. Method: From a study concerning period going from January 2010 to December 2013, the authors attempt to define the rate of cannabis users in the study's population. Next, they seek to describe their social and familial characteristics, and the modality of cannabis use. Finally, they attempt to make correlations between use of cannabis and diagnostic delivered by doctors. Results: $9.13 \%$ of patients who are received during the study's period report personal cannabis use. The middle delay between the first use of cannabis and the first apparition of psychiatric troubles is 4 years and 8 months. The diagnostic of acute delirious episode (BDA in French) is frequently evocated about a first episode or recidivism. Evolution respects classic report of $1 / 3$ (good, bad and indeterminate). Schizophrenia is also evocated in non negligible proportion, particularly in early and prolonged use of cannabis. Conclusion: Psychopathological disorders are frequent among adolescents and young adults who are hospitalised in the psychiatric hospital of Fatick. Use of cannabis in long term seems to provide particularly psychosis.

\section{Keywords}

Drug Addiction; Cannabis; Psychopathological Disorders; Taking into Care; Senegal 


\section{Introduction}

The adolescence is the passage between the childhood and the adulthood, an intermediate generation. The feeling of identity in this period must be distinguished in the one of child or of adult. Emptiness sensation and the resort to changeable identifications are frequent. If the adolescence is often a complex period difficult to live, it is also preceded or accompanied with many other difficulties which the young people try to face in transition to adulthood. So, all kinds of addictions are used by the teenagers to try to facilitate this transition (Bertolini, 2011). Among these, the cannabis seems the most approachable and the most used drug in a lot of countries.

Two main varieties of cannabis are known according to the mode of culture. On the one hand, the cannabis sativa sativa, cultivated on ground, is high-fibber useful for the manufacturing of bags. On the other hand, the cannabis sativa indica, cultivated in hot and dry areas, is rich in basically psychoactive element. It is the product which is more approachable to the young people in our context. Mainly, it is smoked and offers high intensity and duration of the optimal effects because half of the active ingredient is going to be absorbed by this breath way (Phan, Corcos, Girardon, Nezelof, \& Jeammet, 2005). So, the cannabis or Indian hemp is the most popular illicit substance, essentially consumed by inhalation and causes central effects by the minimal dose of $0.05 \mathrm{mg} /$ kg (Llorca, Chereau, Brousse, \& Schawn, 2004).

Some studies concerned in Senegal the use of drugs and their psychosocial consequences, but they are not focused on the cannabis. So, most of them are epidemiological, anthropological or sociological (Werner, 1993; Tine, 2008). In the mental health field, some relatively former studies (Collomb, Diop, \& Ayats, 1962; Gueye, \& Omais, 1983) concerned pathologies associated with drug's addiction. They were especially interested by various used substances, mode of consumption, circuits of the product and various cultural representations. The studies were more global about the age of the users. Then, the teenagers are less representative.

While in the international literature, it tends to be demonstrated that the use of the cannabis at the youngest is a provider of psychopathologic disorders like psychosis or schizophrenia (Krebs, Gut, Plaze, \& Dervaux, 2013), it can be disturbing to see the use of cannabis extending at the minors of age in our country. However, it is necessary to remind that, in an empirical way, the worry of general people concern this possible consequence of drugs and particularly cannabis is already rather former. It based on the frequent observations of psychological or psychiatric disorders about occasional or customary users of cannabis. So, by excess, the parents of the young patient systematically associate any psychotic disorder with the potential use of cannabis or other drugs. We are going to try in this study, from the data stemming from patients' files received and/or followed by the doctors in the mental health hospital "Dalal Xel" of Fatick, to define the rate of the users of cannabis among this population. Afterwards, we attempt to describe their socio-familial characteristics as well as their methods of consumption. On the other hand, we shall try to make correlations between the use of drug and the presented disorders. We shall not finish without making recommendations for the prevention and the struggle against drugs, particularly to the young people.

\section{Methodology}

\subsection{Purpose of the Study}

In this work, the authors try to estimate the position of psycho-toxic substances in the expression and the treatment of the psychopathologic disorders at adolescence, in a mental health hospital, situated in one of the regions inside Senegal, Fatick.

\subsection{Objectives of the Study}

The study articulates around some specific objectives:

- Determine the socio-demographic characteristics of the concerned subjects.

- Determine the average age to the first consumption of cannabis.

- Determine the period between the first consumption and the first appearance of the disorders.

- Identify the various types of substances associated with the consumption of cannabis among the young patients received in the mental health hospital "Dalal Xel" of Fatick from 2010 to 2013.

- Identify their modes of use in this population (ways of administrations, isolated use or use in a group).

- Identify the main diagnostic hypothesis evoked by doctors in front of psychopathologic disorders presented by the young smokers of cannabis received in this hospital. 
- Discuss the impact of the use of cannabis in the happening of later psychiatric disorders among the young people.

- Determine the short and medium-term evolution of the presented psychiatric disorders.

\subsection{Type of Study}

It is about a retrospective, transverse study, in descriptive aim with research for correlation between the use of cannabis among the young people and the occurrence of psychiatric disorders. This study concerns all the patients' files having constituted in the mental health hospital "Dalal Xel" of Fatick.

\subsection{Site of the Study}

The region of Fatick is limited to the North and to the northeast by the regions of Thies, Diourbel and Louga, in the South by the Republic of Gambia, in the East by the region of Kaolack and on the West by the Atlantic

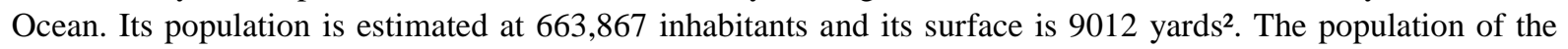
region is relatively young ( $58 \%$ are less than 20 years old). The agriculture, the breeding and the fishing are the main resources. As regards the domains of health and education, the infrastructures are still very limited. Traditional schooling is delivered toward Koran, modern schooling is based on French language. The mental health hospital "Dalal Xel" of Fatick is situated to the left in the entrance of the city of Fatick on the main road from Dakar. Situated 93 miles from Dakar, capital of Senegal, Fatick is a new city distant to 40.3 miles from Mbour (tourism, coastal area) and 27.9 miles from Kaolack (former city with a prosperous business). From the point of view of the institutional organization, Dalal Xel de Fatick is a hospital of catholic obedience, property of "Saint Jean de Dieu" hospital) order and managed by "generous brothers of Saint Jean de Dieu", insuring a department of care in non-profit mental health. It depends on the chief of medical region. The first psychiatric consultations began there in November 2003.

The team consists of:

- The management: 3 "Brothers" manager of the hospital, nurse major, responsible of the drugstore

- One doctor head, psychiatrist

- 08 nurse's aides

- Cleaning operatives

- Gardeners

- Guards.

The main activities are:

- The consultations and ambulatory follow-ups of patients: on Monday, Tuesday and Friday, the Emergency cases;

- The hospitalizations;

- The community consultations in few districts (Diofior, Gossas, Sokone) and at the prison of Fatick: collaboration with the structures of the Ministry of Health.

\subsection{Period of the Study}

The study concerns all the patients' files consulted in the mental health hospital "Dalal Xel” of Fatick, about a period of four years going from January 2010 till December 2013.

\section{Population of the Study}

\subsection{Criteria of Inclusion}

The study concerns at first all the patients of less than 22 years old, who consulted in the hospital between January 2010 and December 2013. Then were selected among them those who revealed, during their hospitalization or during the conversations, a previous repeated use or abuse of narcotics in particular cannabis.

\subsection{Criteria of Exclusion}

The very incomplete or illegible files were excluded. 


\subsection{Studied Variables}

The studied variables will concern the age (years) during the study, the sex (Male, Female), the school level (no schooled, Koran schooled, primary and middle school), the occupation of the patients, the age of men at the first consumption of cannabis (years), the period between the first consumption and the first symptoms (years), the type of use of cannabis (isolated use or use in a group), the mode and the route of drugs administration (oral, inhalation), the main diagnostic hypothesis (according to) the evolution of disorders (favourable, unfavourable).

\subsection{Progress of the Study}

From the target established by all the files brought together over the aforementioned period, a selection of the population of study is made according to the age (21 years in most) then according to the use of one or several narcotics before the consultation in the hospital.

\subsection{Limits of the Study}

The retrospective character of the study confronts with a lot of risk of loss of information. Indeed, in certain files, it misses that one or several information about which we do not know if they were looked for or not. Besides, some criteria as the frequency of the consumption and the quantity of consummate products were not able to be measured because of lack of precision on these data. It's the same as socio-familial conditions. Only the level of the schooling and the professional status of these young adults and big teenagers were considered. What is more, the rarity of the regional psychiatric services makes that the consultations are intended in the mental health hospital "Dalal Xel" of Fatick to a general population essentially dominated by adults. What can make that the youngest children and teenagers can be directed to the Senegalese capital, Dakar, where exist two services of child psychiatry, at the moment. So the frequency of the young people users of cannabis in the consultant population can be widely underestimated.

\section{Results}

On a total of 471 patients' files received in the hospital "Dalal Xel” of Fatick, from January 2010 to December 2013, 43 files (9.13\%) were included in our study and concerned exclusively men.

The following Tables 1-9 highlight the results according to the age of the patients, then according to their age in their first experience of consumption of the cannabis, then according to period between the first experience of consumption of cannabis and the first signs of psychopathologic disorders.

\section{Discussion}

\subsection{Frequent uses of the Cannabis among the Young People}

In Senegal, it seems that the phenomenon of the consumption of narcotics as the cannabis is trivialized by the populations and the families even if the State proposes laws which penalize and condemn severely the holders,

Table 1. Distribution according to the age.

\begin{tabular}{cccc}
\hline Age (years) & Quantity & Relative frequencies (\%) & Accumulated frequencies (\%) \\
\hline 17 & 1 & 2.3 & 2.3 \\
18 & 8 & 18.6 & 20.9 \\
19 & 4 & 9.3 & 30.2 \\
$\mathbf{2 0}$ & $\mathbf{1 8}$ & $\mathbf{4 1 . 9}$ & $\mathbf{7 2 . 1}$ \\
21 & 12 & 27.9 & 100 \\
Total & 43 & 100 & \\
\hline
\end{tabular}

Minimum: 17; Maximum: 21; Average: 19.7; Mode: 20. The average age of the patients is of 19.7 years. The age of 20 years represents the one which the patients are reached most of the time by psychiatric disorders in our sample. But 18 and 21 years were other peaks of frequency. 
Table 2. Distribution according to the period between the first consumption and the first symptoms, and the age of the patients.

\begin{tabular}{|c|c|c|c|c|c|c|c|c|c|c|c|c|c|}
\hline & $\begin{array}{l}\text { Period between the } \\
\text { first consumption and } \\
\text { the first symptoms }\end{array}$ & Unknown & $<1$ & 1 & 2 & 3 & 4 & 5 & 6 & 7 & 13 & After & Total \\
\hline \multicolumn{14}{|c|}{ Age (years) } \\
\hline 17 & & 1 & 0 & 0 & 0 & 0 & 0 & 0 & 0 & 0 & 0 & 0 & 1 \\
\hline 18 & & 3 & 2 & 1 & 1 & 0 & 1 & 0 & 0 & 0 & 0 & 0 & 8 \\
\hline 19 & & 1 & 2 & 0 & 0 & 0 & 0 & 0 & 0 & 0 & 0 & 1 & 4 \\
\hline 20 & & 8 & 2 & 3 & 1 & 1 & 0 & 0 & 2 & 0 & 1 & 0 & 18 \\
\hline 21 & & 5 & 1 & 1 & 2 & 1 & 0 & 1 & 0 & 1 & 0 & 0 & 12 \\
\hline Total & & 18 & 7 & 5 & 4 & 2 & 1 & 1 & 2 & 1 & 1 & 1 & 43 \\
\hline
\end{tabular}

Minimum: <1; Maximum: 13; Average: 4.8. The period between first consumption and first symptoms of psychiatric disorders is lower than one year or 2-year-old for more than half of cases, so be it 16 on 25 who gave the information about this datum.

Table 3. Distribution according to the age of the men at the first consumption of cannabis.

\begin{tabular}{cccc}
\hline $\begin{array}{c}\text { Age of men at the first } \\
\text { consumption of cannabis } \\
\text { (years) }\end{array}$ & Quantity & $\begin{array}{c}\text { Relative frequencies } \\
\text { (\%) }\end{array}$ & $\begin{array}{c}\text { Accumulated frequencies } \\
\text { (\%) }\end{array}$ \\
\hline Unknown & $\mathbf{1 9}$ & $\mathbf{4 4 . 2}$ & $\mathbf{4 4 . 2}$ \\
13 & 2 & 4.7 & 48.8 \\
14 & 2 & 4.7 & 53.5 \\
15 & 3 & 7 & 60.5 \\
16 & 2 & 4.7 & 65.1 \\
17 & 2 & 4.7 & 69.8 \\
$\mathbf{1 8}$ & $\mathbf{7}$ & $\mathbf{1 6 . 3}$ & $\mathbf{8 6}$ \\
19 & 5 & 11.6 & 97.7 \\
20 & 1 & 2.3 & 100 \\
\hline
\end{tabular}

Minimum: 13; Maximum: 20; Average: 16.9; Mode: 18, 18 on 24 patients are 3/4 declared to have had their first consumption in 18 years or previously. A big part of the meditative data does not state this datum, so be it 19/43.

Table 4. Distribution according to the use in a group.

\begin{tabular}{lccc} 
& Yes & No & Total \\
\hline Use in a group & 39 & 4 & 43 \\
\hline
\end{tabular}

The overwhelming majority of the patients declare a use of cannabis in groups of companions (39/43), but also sometimes in an isolated way.

Table 4b. Distribution according to the isolated use.

\begin{tabular}{cccc} 
& Yes & No & Total \\
\hline Isolated use & 22 & 21 & 43 \\
\hline
\end{tabular}

As regards the isolated use, approximately half of the sample (21/43) is not confronted with it. 
Table 5. Distribution according to the main consummate product.

\begin{tabular}{ccc}
\hline Consummate products & Quantity & Relative frequencies \\
\hline Alcohol & 1 & 2.3 \\
Cannabis & $\mathbf{4 0}$ & $\mathbf{9 3}$ \\
Organic solvents & 2 & 4.7 \\
Total & 43 & 100 \\
\hline
\end{tabular}

The cannabis is the product most frequently used (40/43) sometimes in association with the alcohol. Solvents were rarely used (2/43) and in an exclusive way. One patient drinks some alcohol without other consumption of toxin.

Table 6. Distribution according to the main diagnostic hypothesis.

\begin{tabular}{ccc}
\hline Diagnostic hypothesis & Quantity & Relative frequencies \\
\hline None & 2 & 4.7 \\
Obsessive access & 1 & 2.3 \\
Acute delirious in first access & $\mathbf{2 3}$ & $\mathbf{5 3 . 5}$ \\
Acute delirious in recurrent episodes & $\mathbf{7}$ & $\mathbf{1 6 . 3}$ \\
Depression & 1 & 2.3 \\
Epilepsy & 1 & 2.3 \\
Schizophrenia & 7 & 16.3 \\
Anxious disorders & 1 & 2.3 \\
Total & 43 & 100 \\
\hline
\end{tabular}

The diagnostic hypothesis most frequently advanced is the acute delirious in first access (23/43) or in recurrent episodes (7/43); then comes the schizophrenia (7/43).

Table 7. Distribution according to the evolution.

\begin{tabular}{ccc}
\hline & Quantity & Relative frequencies \\
\hline Evolution & 13 & 30.2 \\
Unknown & $\mathbf{1 6}$ & $\mathbf{3 7 . 2}$ \\
Favourable & 14 & 32.6 \\
Unfavourable & 43 & 100 \\
Total & & \\
\hline
\end{tabular}

The evolution is considered as favourable in one more than third party of the cases (16/43). For every other third party, it is indistinct (13/43) or openly unfavourable (14/43).

Table 8. Distribution according to the level of studies of the patients.

\begin{tabular}{ccc}
\hline Level of studies & Quantity & Relative frequencies \\
\hline Unknown & 10 & 23.3 \\
Middle school & $\mathbf{1 2}$ & $\mathbf{2 7 . 9}$ \\
Koran school & 7 & 16.3 \\
Primary school & 8 & 18.6 \\
No schooled & 6 & 13.9 \\
Total & 43 & 100 \\
\hline
\end{tabular}

The level of studies of the patients is ill-assorted with a majority (12/43) which reached the middle school. But a large part of files does not state this datum (10/43). 
Table 9. Distribution according to the occupation of the patients.

\begin{tabular}{|c|c|c|}
\hline Occupations & Quantity & Relative frequencies \\
\hline Nothingness & 16 & 37.2 \\
\hline Apprentice & 1 & 2.3 \\
\hline Shepherd & 1 & 2.3 \\
\hline Car driver & 1 & 2.3 \\
\hline Unemployed & 6 & 14 \\
\hline Trading & 2 & 4.7 \\
\hline Farmer & 3 & 7 \\
\hline Electrician & 1 & 2.3 \\
\hline Primary school teacher & 1 & 2.3 \\
\hline Gardener & 1 & 2.3 \\
\hline Mason & 2 & 4.7 \\
\hline Joiner & 1 & 2.3 \\
\hline Motorcycle Taxi man & 1 & 2.3 \\
\hline Welder & 1 & 2.3 \\
\hline Tailor & 2 & 4.7 \\
\hline Worker & 1 & 2.3 \\
\hline Pupil & 2 & 4.7 \\
\hline Total & 43 & \\
\hline
\end{tabular}

A very big part of files states an absence of profession (16) or unemployed person (6), which are in all 22 on 43 concerned patients. Others are limited to jobs in tertiary sector and are essentially apprentices.

the users and the traffickers of these products. Werner already indicated the trend of the mothers to keep silent about the use of narcotics by their son because of the Senegalese "sutura" (Werner, 1993). Today still, it is in houses and popular districts that are in hiding the biggest traffickers. Some young people smoke the cannabis joint in the middle of the street, in front of their relatives or their friends, without fear of being reprimanded or denounced. The latter insure them a good cover in case of police intervention. It is a kind of everyday acceptance which could explain the increasing expansion of the consumption of cannabis, particularly among the young people. It associates all other socio-cultural factors (Werner, 1993) which surround the consumption of drugs, in particular the myths, the mystic practices (ritual, initiatory) and the ambivalent positions (religious tolerance, the taste for danger, the pleasure, "poisons" or "remedies"). The role of drugs in the link of sociality always existed placing them sometimes as intensifier, sometimes as destroyer of this link. So, the cannabis smoked in several can have a socializing role in adolescence (Wojciechowski, 2005) and intensifier of the identity of group of the teenagers or the young adults. Some studies show that the family situation appears as a not insignificant factor, in particular the situations of separation, divorce or death in the parental couple (Tine, 2008). It also likes the societal changes which are more and more important weighing down the work of adolescence. So, some data state a more and more important number of minors arrested by the police, as for example in publications of Ocrtis (central office) for the repression of the illicit traffic of narcotics): during the period from 1999 till 2002, the increase of number was more than ten times (Tine, 2008).

\subsection{The Position of the School}

In the adolescence, the school could represent a good barometer to estimate the good health of the young people. Is relatively stiff frame confronts them with a duty of adaptation which requests regularly the flexibility of their 
intellectual and emotional abilities. In this way, some considered the school as the producer of morons and social misfits by its rather stiff frame. In our population of study, the young people are removed from school for the greater part, but a good part reached the middle school. However, none of them reached the university level. The educational delay must be however put in relation also with the relatively weak level of the conditions of schooling in regions outside the capital. Except it and considering some brilliant results obtained by some pupils in the same context, it is justified to wonder to what of other factor is bound this academic failure. Isn't it a consequence due to the consumption of cannabis? Wouldn't the school difficulties have facilitated the consumption of cannabis? Aren't there factors which can induce as well school problems as the consumption of cannabis? The answers to these questions can be complex. However, it is necessary to note that the intellectual curiosity which requires the process of learning also joins to the tendency to act it and in the risk-takings, in the adolescence. It can so appear as being obvious that an intelligent enough young person experiments a product considered as dangerous or able to produce fabulous effects. But it is not all the young people who hang on to the product, becoming addict. It evokes inevitably other factors among which those emotional and social factors. The school remains as such a mattering frame to strengthen or straighten the evolutionary processes. In every case, the school remains inevitably a factor mattering for the young people in school age. Then, some authors (Ndiaye, 2003) defend the idea that the school is a factor which would favour the failure of the socialization, because of the drug addiction abnormality. For our part, it is difficult to put things in perspective between what the school leads at a young person's until he becomes a drug addict and what the drug addiction induces to a pupil in term of disorders of the school adaptation or the learning difficulties. Scholar failure or unhooking is possible. The period of adolescence which corresponds more or less to the access to the middle school must not be neglected, considering all the changes produced by this new status of the teenager.

The authority of the primary schoolteacher seems dissolved in front of the multiplication of the teachers who follow one another in a class of middle school. The discovery of new relational modalities in the school field offers at the same time more and spread possibilities in the process of subjectivation but also exposes to the separation from stable identification's figures. It is all the more heavy as the social and family environment offers fewer marks. Indeed, judging effects of the post-modern environment on the process of subjectivation, Lazartigues finds that the authority and its double, the obedience, are reduced to the requirements of school learning and protection (Lazartigues, 2013).

\subsection{Diagnostic and Evolutionary Aspects}

The hypothesis of acute delirious episode (BDA) was most frequently put forward in the files of our study. She can be put closer to the form of schizophrenia disorders in the DSM-IV, the acute and passing psychotic disorders of the international classification of the diseases (CIM-10) and the acute psychosis of the CFTMEA. In the classic French nosology, the BDA includes all the psychotic pathologies of duration 6-month-old subordinate and thus includes disorders varied on the unclear borders. This limit establishes at the same time its interest of the concept. The advantage of this concept at the adolescence is not to anticipate the place of this syndrome in the spectre of the schizophrenia (Botbol, Barrère, \& Speranza, 2004). So, the BDA is an entity which answers the assertion of a psychopathologic fact, integrating the body, the history of the subject and the experience of his environment, by maintaining opened the question of its psychopathology (Weibel \& Metzger, 2005).

Besides, the diagnostic hypothesis of acute delirious episode (CFTMEA) goes with the notion of psychosis inferred by a substance (DSM), in particular when the disorders owed less than a month. But in other cases and because of a significant number of losses of follow-ups, the diagnosis cannot be retained for a great majority of the patients of our population of study. Weibel and Metzger quoting Baubet and Moro remind that "the diagnosis of delirious episode keeps all its interest”, not auguring either of the meaning of the episode, nor of the forecast, being able to correspond to an attempt of identity reorganization (Weibel \& Metzger, 2005). Indeed, if the acute psychotic episode translates fragility, it also expresses plasticity by its variable future according to the care and treatment, putting in perspective the exercise of prediction (Weibel \& Metzger, 2005).

In theory, we can distinguish the use, the abuse and the dependence of cannabis. But in practice, it turns out more complex, particularly in the fall of an psychopathological episode to put things in perspective. It is necessary to recognize that the risk of the cannabis drunkenness induces modifications of sensory perception, changes of the memory, disorders of the awareness and behaviour disorders. Called also "bad trip", it can be in the form of fit of panic, of paranoiac feelings, of hallucinations or delirious. It is often the case of overdoses which can 
cause, besides the hallucinations and besides the delirious, disorders of the heart rhythm, aggressiveness as well as road accidents or the work accidents (Llorca, Chereau, Brousse, \& Schawn, 2004). These incidents are the main motives for consultation or for hospitalization and can represent the only opportunity to highlight the noxiousness of the product. In the uses (or misuses) in the long term, the unmotivational syndrom is classically described, but meets hardly in our context, by the fact certainly of a very high unemployment rate in the population.

The consumption of drugs and in particular cannabis is often associated in a empirical way with the appearance of the mental illness, to a large number of our fellow countrymen. It goes with a certain fear of a use not regulated culturally. Indeed, the cannabis is not considered as dangerous in the orderly frame of ritual practices (Suissa, 1996; Fourré, 2009-2010), but its use outside this frame seems to expose the individuals and the groups to its negative effects, to the disease and to the madness. However, no study on the national level comes to confirm or to counter this observation. If besides, some authors (Glauser \& Thievent, 2004) find that the cannabis does not most probably infer chronic psychoses persisting except the periods of consumption, others (Krebs, Gut, Plaze, \& Dervaux, 2013) highlight the toxicity of the cannabis at the young teenagers in particular with a risk important to develop in the adulthood psychosis or schizophrenia.

In spite of the frequency of the psychiatric disorders among the teenagers who have used cannabis, it will be necessary to avoid reducing the problem to the medical or psychiatric field. Indeed, all the drug addicts neither are psychotic, nor even psychiatric and the phenomenon of the use of drugs or addiction is multidimensional (Phan, 2005). However, many studies showed that the moderate consumption even minimal quantity of cannabis established a risk factor mattering in the occurrence of later psychotic disorders. That is especially true if the subject was young, adolescent (in period of intellectual maturation), affected by schizo-typical personality, subclinical psychotic symptoms preliminary or personal histories of sexual abuse, or psychotic disorders in his family, or that the rates of psychoactive cannabinoïdes are raised in the samples of consummate cannabis. But there is still no evidence that the schizophrenic evolution is directly and exclusively bound to it. The consequences of the consumption of cannabis also contain the anxious disorders, the early school leaving, the lack of motivation and the difficulties of insertion (Krebs, Gut, Plaze, \& Dervaux, 2013).

It is clear, in some situations, that subjects very disrupted on the mental health field are engaged for diverse reasons in the consumption of cannabis. In this case, the noxious effects of the cannabis at a person presenting schizophrenia are recognized particularly if he was following a chronic exposure. Indeed, psychotic disorders more patent, luck of attention, global cognitive deficit and a decrease of volume in some regions of the brain, in particular the prefrontal brain, were found (Glauser \& Thiévent, 2004; Krebs, Gut, Plaze, \& Dervaux, 2013). The variability of the clinical evolution imposes, in every case, the stop of the consumption which is justified and facilitated by the care associating an adapted chemotherapy and behavioural approaches (Krebs, Gut, Plaze, \& Dervaux, 2013). In this context, the motivational therapies and the psycho-education facilitate the stop of the consumption and strengthen the therapeutic alliance (Krebs, Gut, Plaze, \& Dervaux, 2013).

\subsection{Socio-Familial Conditions}

The users of cannabis, in our population of study, are a matter of socioeconomic circles highly varied. If it is usual to consider that the drug addiction is a plague of cities and shanty towns, it is necessary to recognize that in our campaigns the users are not so rare. The conditions of precariousness which were generally recognized as favouring factors are shared today better and better by the campaigns which become distorted. As such Fatick is relatively a campaign such a new city which groups urban and rural characteristics. So, some patients living in Dakar have some family relationships with autochthons and take advantage of opportunities of one of the rare institutions of the country. It can make more complex the interpretation of the data as for the rural life of some aspects. According to the available data, the disparity of the population of the users seems confirm. However, it is necessary to find their failure or frequent early school leaving, particularly from the middle school. What confronts them, despite of an apparently normal intelligence, to a limited choice for occupation and establish one of the most dramatic consequences of their socio-familial and professional insertion.

\section{Recommendations}

The increasing of the consumption of cannabis in the young people in our country is a real problem of public health and a important brake for the development. To face it, it is not only essential to strengthen the policy of 
prevention and existing care, but especially to take into account the particular aspect of the development of the young people and around the period of the adolescence. The interest to emphasize the prevention regarding consumption at the young teenagers bases on the higher risk of abuse and/or dependence, of polyconsommation and passage to other illicit drugs with a sanitary cost brought up to the adulthood (Phan, Corcos, Girardon, Nezelof, \& Jeammet, 2005). In this goal, it is necessary to have multidisciplinary teams combining at the same time sanitary and social approach, practices of networks by avoiding the confusions and the substitutions among professionals (Pelège, 2012). The discomfort of the young people who get in touch with drugs and in particular the cannabis is well known. Their feeling of isolation and the need to be accompanied by friendly, secure and rigorous adults are not still expressed, otherwise by acting out which feed the conflicts with the society. The contribution of the adults in environment around the adolescent is essential to mediatise the social relationship and facilitate the elaboration and the overtaking of the intra-psychic conflicts. The rashness or boldness of the adolescent is a response to the need for recognition, for legitimacy, for support, for understanding of the suffering of the young person (Le Breton, 2013). The approach can however be delicate especially if we take into account risks of narcissistic wounds of the young person confronted to recognize explicitly his position of weakness (Matot, 2012). In every case, it seems sensible to question on the possibilities of solutions being able to dread the case of the teenager not in its only addicting dimension but in its entirety, its global nature (Bertolini, 2011).

\section{References}

Bertolini, M. (2011). Adolescence et Addictions. Revue Médicale Suisse, 7, 1794-1798.

Botbol, M., Barrère, Y., \& Speranza, M. (2004). Psychoses à l'adolescence. EMC-Pédiatrie, 1, 259-270.

Collomb, H., Diop, M., \& Ayats, H. (1962). Intoxication par le chanvre indien au Sénégal. Cahiers d'Etudes africaines, III. 9, 139-144.

Fourré, M. (2009-2010). Les enfants de parents drogués. Psychopathologie Africaine, XXXX, 159-175.

Glauser, J., \& Thiévent, R. (dir. Hainard, F.) (2004). Jeunesses, cannabis et autres addictions: quelles préventions? Mandat du bureau de la commission addiction en réponse à la motion déposée au Grand Conseil neuchâtelois par M (142 p). Neuchâtel: Bernard Matthey Institut de sociologie-Université de Neuchâtel.

Gueye, M., \& Omais, M. (1983). Tentative pour une approche socioculturelle de l’usage abusif des drogues au Sénégal. Psychopathologie Africaine, XIX, 141-172.

Krebs, M.-O., Gut, A., Plaze, M., \& Dervaux, A. (2013). L’impact du cannabis à l'adolescence sur la transition psychotique de l'adulte. Neuropsychiatrie de l'Enfance et de l'Adolescence, 61, 224-230.

Lazartigues, A. (2013). De l'enfance à l'âge adulte: Quelques effets d'un environnement postmoderne sur le processus de subjectivation. Neuropsychiatrie de l'Enfance et de l'Adolescence, 61, 302-309.

Le Breton, D. (2013). Adolescences en Difficulté (pp. 24-30). VST_Vie Sociale et Traitements.

Llorca, P.-M., Chereau, I., Brousse, G., \& Schawn, R. (2004). Troubles psychiatriques d'origine toxique ou alimentaire. EMC-Psychiatrie, 1, 188-200.

Matot, J. (2012). L'enjeu adolescent. Déconstruction, enchantement et appropriation d'un monde à soi (352 p). Paris: PUF.

Ndiaye, L. (2003). L’enfance, rapport entre cultures et déviances. In Les cahiers histoire et civilisations (pp. 141-151). Juillet: UCAD.

Pelège, P. (2012). Un point de vue sociologique sur la question addictive (pp. 24-29). Revue VST-Vie Sociale et Traitements.

Phan, O. (2005). La prise en charge des consommations de cannabis à l'adolescence. Psychotropes, 11, 53-63.

Phan, O., Corcos, M., Girardon, N., Nezelof, S., \& Jeammet, P. (2005). Abus et dépendance au cannabis à l'adolescence. EMC-Psychiatrie, 2, 207-224.

Suissa, A. J. (1996). Culture, santé mentale et toxicomanie. In Toxicomanie et santé mentale: les actes du XXIVe colloque, édité par l'Association des Intervenants en Toxicomanie du Québec (pp. 118-128). Montréal: l'Association des Intervenants en Toxicomanie du Québec.

Tine, B.(2008). La toxicomanie: étude sociologique d'une forme de déviance au Sénégal (214 p). Paris: EPU.

Weibel, H., \& Metzger, J.-Y. (2005). Psychoses délirantes aiguës. EMC-Psychiatrie, 2, 40-61.

Werner, J.-F. (1993). Approche ethnographique de l’usage des drogues au Sénégal. Psychopathologie Africaine, XXV, 323345.

Werner, J.-F. (1993). Urbanisation et déviance. Études anthropologiques sur la drogue au Sénégal. Cahiers des. Sciences 
Humaines, 29, 3-32.

Wojciechowski, J.-B. (2005). Pratiques médicales et usages de drogues: linéaments de la construction d’un champ. Psychotropes, 11, 179-207. 